\title{
Evapotranspiração de referência em Januária-MG pelos métodos tanque classe "A" e Hargreaves-Samani
}

\author{
Eliane Ramos Oliveira ${ }^{1}$, Tatiane Carla Silva ${ }^{2}$, Rogério Ferreira de Oliveira Ramos ${ }^{1}$ \\ ${ }^{1}$ Instituto Federal do Norte de Minas Gerais - IFNMG. ${ }^{2}$ Universidade Federal de Lavras, Lavras, MG. E-mail: \\ tcs.agronomia@gmail.com
}

\section{Resumo}

Objetivou-se com este trabalho avaliar o desempenho do método do tanque classe " $A$ " (TCA) e HargreavesSamani (HS), comparados ao método de Penman-Monteith (PM), para determinação da evapotranspiração de referência (ETO) no município de Januária, MG. Foram utilizados os dados climáticos do período 01 de janeiro de 2014 a 31 de dezembro de 2016, pertencente ao banco de dados da estação meteorológica do INMET, localizada no município. A partir dos dados obtidos foram realizadas análises de correlação entre os métodos TCA e HS com o PM, de regressão linear e determinação dos índices de concordância de Willmott e de desempenho. De acordo com os resultados obtidos, pode-se concluir que o método do TCA superestima a ETO na região de Januária-MG, mas teve desempenho bom quando ajustado ao método de PM. Quanto ao método de HS, os resultados obtidos mostram que este superestimou a ETO na maioria dos meses, porém apresentou coeficiente de correlação alto e desempenho satisfatório, sendo assim, ambos os métodos podem ser utilizados para estimar a ETO na região de Januária MG, quando não se dispuser de todos os dados necessários para determinação por meio do método de PM.

Palavras-chave: manejo de irrigação; Peman-Monteith; agroclimatologia.

\section{Evapotranspiration estimation in Januária-MG by class " $A$ " pan and Hargreaves-Samani methods}

\begin{abstract}
The objective of this work was to evaluate the performance of the class " $A$ " (TCA) and Hargreaves-Samani (HS) method, compared to the Penman-Monteith (PM) method, to determine the reference evapotranspiration (ETO) in Januária, MG. We used the weather data from January 1, 2014 to December 31, 2016 , belonging to the database of the INMET weather station, located in the municipality. From the obtained data, correlation analyzes between the TCA and HS methods with the PM, linear regression and determination of the Willmott agreement and performance indices were performed. According to the results obtained, it can be concluded that the ACT method overestimates the ETO in the region of JanuariaMG, but performed well when adjusted to the PM method. As for the HS method, the results show that it overestimated the ETO in most months, but presented a high correlation coefficient and satisfactory performance, so both methods can be used to estimate the ETO in the region of Januária MG, when not having all the data necessary for determination by the PM method.
\end{abstract}

Keywords: irrigation management; Peman-Monteith; agroclimatology.

\section{Introdução}

O planejamento e aproveitamento da água na produção agrícola, bem como o desenvolvimento de metodologias que permitam estimar o correto volume de água a ser aplicado, são de fundamental importância econômica e para a preservação da disponiblidade dos recursos hídricos (LUNARDI; RABAIOLLi, 2013).

Em tempos de escassez, é fundamental a discussão de planos alternativos, e principalmente eficazes, de redução do consumo e desperdício da água (AUGUSTO et al., 2012). 
Qualquer planejamento de um projeto de irrigação que vise à máxima produção e à boa qualidade do produto usando de maneira eficiente a água, requer conhecimentos das interrelações entre solo-água-planta-atmosfera e manejo de irrigação (KUNZ et al., 2014). Cerca de $70 \%$ da quantidade de água precipitada sobre a superfície terrestre retorna à atmosfera pelos efeitos da evaporação e transpiração. Devido isso, a quantificação desses dois processos é de fundamental importância para realização de projetos de irrigação. O manejo de irrigação requer em seu planejamento a determinação da quantidade de água necessária, tendo como um dos principais componentes a evapotranspiração (MIRANDA et al., 2010).

A evapotranspiração é o processo por meio do qual a vegetação e o solo enviam umidade para a atmosfera, aumentando a umidade do ar, tornando as chuvas mais constantes (ALENCAR et al., 2009). A definição da evapotranspiração (ETO) é de fundamental importância, pois define o consumo de água pelas plantas e, por consequência, a lâmina de irrigação a ser aplicada pelo sistema.

Existem vários métodos que são usados para estimar a evapotranspiração e são baseados em dados meteorológicos. A maior parte deles é de difícil aplicação, não só pela complexidade do cálculo, mas por exigirem grande número de elementos meteorológicos, somente fornecidos por estações de primeira classe ou automáticas. Neste sentido, destaca-se o método PenmanMonteith (PM), considerado como o método padrão pela FAO pela sua precisão e excelentes resultados nas mais distintas condições climáticas. São empregados na sua estimativa dados de temperatura $(T)$, umidade relativa do ar (UR), radiação solar (RS) e velocidade do vento (V). Os produtores rurais, contudo, nem sempre dispõem desses dados, sendo necessária a utilização de métodos mais simples para o cálculo de ETO (BERNARDO, 2019).

O evaporímetro tanque classe " $\mathrm{A}$ ", em virtude do custo relativamente baixo e do fácil manejo, tem sido empregado nos projetos de irrigação. Ele tem a vantagem de medir a evaporação de uma superfície de água livre, associada aos efeitos integrados da radiação solar, do vento, da temperatura e da umidade do ar (BERNARDO, 2019). A ETO neste caso é calculada multiplicando-se os valores diários da evaporação do tanque (ECA) por um coeficiente (Kp), em função da distância da bordadura ao redor do tanque, da velocidade do vento $(V)$ e da umidade relativa do ar (UR) (ESTEVES et al., 2010)

Métodos que utilizam somente a temperatura do ar para a estimativa da ETO também podem ser empregados pelos produtores para o manejo da irrigação, como o método Hargreaves e Samani (PILAU et al., 2012). O método vem sendo utilizado amplamente devido a sua simplicidade e potencialidade para calibração de seus parâmetros, sendo uma alternativa viável para a estimativa ETO quando não se tem as informações dos parâmetros climáticos exigidos pelo método PM. Nesse modelo necessita-se apenas das temperaturas mínima, máxima, média do ar (BAUTISTA et al., 2009).

No município de Januária, localizado no norte de Minas Gerais, são escassos os trabalhos sobre o uso racional da água. A avaliação de métodos como o HS e TCA pode resultar em equações de ajuste local, que poderão auxiliar no planejamento e gerenciamento de irrigação na região, principalmente por parte dos pequenos irrigantes, que não têm acesso aos dados meteorológicos necessários para determinação da ETO pelo método de PM.

Nesse sentido, o presente trabalho teve como objetivo comparar os valores diários de ETO determinados pelo método de PenmanMonteith-FAO com os valores estimados pelos métodos do tanque Classe A e de Hargreaves \& Samani na região de Januária, norte de Minas Gerais.

\section{Material e Métodos}

O trabalho foi realizado no Instituto Federal de Educação Tecnológica do Norte de Minas Gerais, localizado no município de Januária, Minas Gerais, a $15^{\circ} 26^{\prime}$ de latitude Sul e $44^{\circ} 22^{\prime}$ de longitude Oeste, com temperatura média anual de $24,4{ }^{\circ} \mathrm{C}$. Para a realização deste estudo, foram utilizados dados meteorológicos diários de temperaturas (máxima, média e mínima), umidade relativa, radiação solar recebida $e$ velocidade do vento de uma série de 3 anos, compreendida em 01 de janeiro de 2014 à 31 de dezembro de 2016, obtida do banco de dados da estação climatológica localizada no Instituto Federal do Norte de Minas Gerais (FNMG), campus Januária, cedidos, para fins de estudo, pelo Instituto Nacional de Meteorologia (INMET).

A evapotranspiração de referência (ETO), foi determinada pelo método do tanque classe " $\mathrm{A}$ ", por meio da Equação 1 proposta por (Bernardo, 
2019) utilizando os dados de evaporação média em $(\mathrm{mm})$; velocidade do vento $\left(\mathrm{m} \mathrm{s}^{-1}\right)$; umidade relativa (\%).

$$
\mathrm{ETO}=\mathrm{EVXKT}(1)
$$

Em que:

ETO = evapotranspiração de referência, $\mathrm{mm} / \mathrm{dia}$

$\mathrm{EV}=$ evaporação do Tanque Classe $\mathrm{A}$ em $\mathrm{mm} / \mathrm{dia}$

$\mathrm{KT}=$ coeficiente de correção, adimensional.

Os valores de Kt serão determinados pela equação 2 proposta por (Bernardo, 2019). es - ea $=$ deficit de pressão de vapor de saturação, em kpa;

$\Delta=$ declividade da curva de pressão de vapor de saturação, temperatura, $\mathrm{kpa}^{\circ} \mathrm{C}^{-1}$;

$\gamma=$ constante psicométrica, $\mathrm{kpa}^{\circ} \mathrm{C}^{-1}$;

A determinação da evapotranspiração de referência (ETO) pelo método de HargreavesSamani foi feita utilizando os dados: temperatura (máxima; média e mínima), e radiação solar usando a equação 4 proposta por (Silva et. al. 2005).

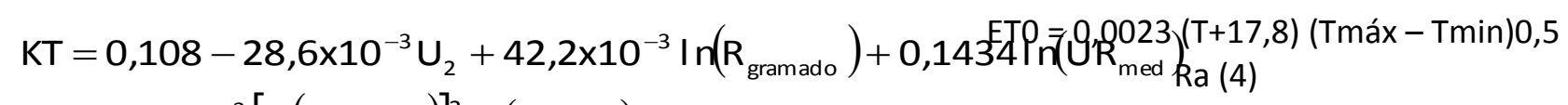

$-0,631 \times 10^{-3}\left[\operatorname{In}\left(R_{\text {gramado }}\right)\right]^{2} \operatorname{In}\left(U R_{\text {med }}\right)$

Em que:

$\mathrm{U} 2$ = é a velocidade média diária do vento a $2 \mathrm{~m}$ de altura, $\mathrm{m} \mathrm{s}-1$

UR = umidade relativa media, $\%$.

$\mathrm{R}_{\text {gramado }}=$ menor distancia do centro do tanque ao limite da bordadura, em metros.

A determinação da evapotranspiração de referência (ETO) pelo método de PenmanMonteih, tido como padrão, foi realizada utilizando os dados: temperatura (máxima, média e mínima), velocidade do vento $\mathrm{m} \mathrm{s}^{-1}$; umidade relativa e radiação solar usando a equação 1 , proposta por Allen et al. (1998).

ETO $=\frac{0,408 \Delta\left(R_{n}-G\right)+\gamma 900 /\left(\frac{900}{T+273}\right) U_{2}\left(e_{s}-e_{a}\right)}{\Delta+\gamma\left(1+0,34 U_{2}\right)}$

Em que:

ETO = evapotranspiração (ETO) de referencia, $\mathrm{mm}$ $\mathrm{d}^{-1}$

$\mathrm{Rn}=$ radiação liquida na superfície da cultura, $\mathrm{Mj}^{-}$ ${ }^{1} \mathrm{~m}^{-2} \mathrm{~d}^{-1}$

$\mathrm{G}=$ densidade do fluxo de calor do solo, $\mathrm{Mj}^{-1} \mathrm{~m}^{-2} \mathrm{~d}^{-}$ 1 ;

$\mathrm{T}=$ temperatura do ar a $2 \mathrm{~m}$ de altura, em ${ }^{\circ} \mathrm{C}$;

$\mathrm{U}_{2}=$ velocidade do vento a $2 \mathrm{~m}$ de altura, em $\mathrm{m} \mathrm{s}^{-}$

$1 ;$

es = pressão de vapor de saturação, em kpa;

ea = pressão atual de vapor, em kpa;
Onde:

ETO $=$ evapotranspiração de referência $(\mathrm{mm}$ dia $\left.{ }^{1}\right)$;

$\mathrm{T} \quad=$ temperatura média do ar a $2 \mathrm{~m}$ de altura $\left({ }^{\circ} \mathrm{C}\right)$;

Tmáx = temperatura máxima do ar a $2 \mathrm{~m}$ de altura $\left({ }^{\circ} \mathrm{C}\right)$;

Tmin = temperatura mínima do ar a $2 \mathrm{~m}$ de altura $\left({ }^{\circ} \mathrm{C}\right)$; e

$\mathrm{Ra}=$ radiação extraterrestre ( $\mathrm{mm}$ dia-1).

Os valores estimados de ETO por HS e TCA

foram comparados com os de PM utilizando-se regressão linear, obtendo-se assim o coeficiente de determinação $\left(R^{2}\right)$.

Foi determinado o coeficiente de exatidão (d), proposto por WILLMONT et al (1985), descrito por Camargo \& Sentelhas (1997) apud Conceição \& Mandelli (2005), através da equação 5.

$$
\mathrm{d}=1-\left\{\frac{\sum(\mathrm{Pi}-\mathrm{Oi})^{2}}{\sum(|\mathrm{Pi}-\mathrm{O}|+|\mathrm{Oi}-\mathrm{O}|)^{2}}\right\}
$$

Onde:

$d$ = coeficiente de exatidão;

$\mathrm{Pi}=$ Valores estimados;

$\mathrm{Oi}=$ Valores obtidos por PM; e

$\mathrm{O}=$ Média dos valores de PM.

Também foi utilizado o coeficiente de desempenho (c), obtido por meio da multiplicação do coeficiente de correlação $(r)$ pelo coeficiente de exatidão (d). 
Tabela 1. Classificação dos métodos de estimativas de acordo com o coeficiente (c).

\begin{tabular}{|c|c|}
\hline Valores do coeficiente de desempenho "c" & Desempenho \\
\hline (5) & Ótimo \\
\hline $0,76-0,85$ & Muito bom \\
\hline $0,66-0,75$ & Bom \\
\hline $0,61-0,65$ & Mediano \\
\hline $0,51-0,60$ & Sofrível \\
\hline $0,41-0,50$ & Mal \\
\hline$\leq 0,40$ & Péssimo \\
\hline
\end{tabular}

Fonte: Camargo e Sentelhas (1997).

Foi realizado o teste $t$ (Student) para determinar a significância estatística usando-se o software SAEG, todos os demais cálculos foram realizados com o auxílio do software Microsoft Office Excel.

\section{Resultados e Discussão}

$\mathrm{Na}$ Figura 1, apresenta-se os valores médios mensais da ETO, em $\mathrm{mm} \mathrm{dia}^{-1}$, no período de 01 de janeiro de 2014 a 31 de dezembro de 2016, correspondentes ao método de Hargreaves-Samani, comparado ao método padrão Penman-Monteith parametrizado pela FAO.

Figura 1. (a) Modelo de regressão ajustado para ETO estimada por PM e a ETo estimada por HS, referente ao período de 01 de janeiro de 2014 a 31 de dezembro de 2016; (b) Comparação entre a ETo estimada por PM e a ETo estimada por HS, referente ao período de 01 de janeiro de 2014 a 31 de dezembro de 2016.
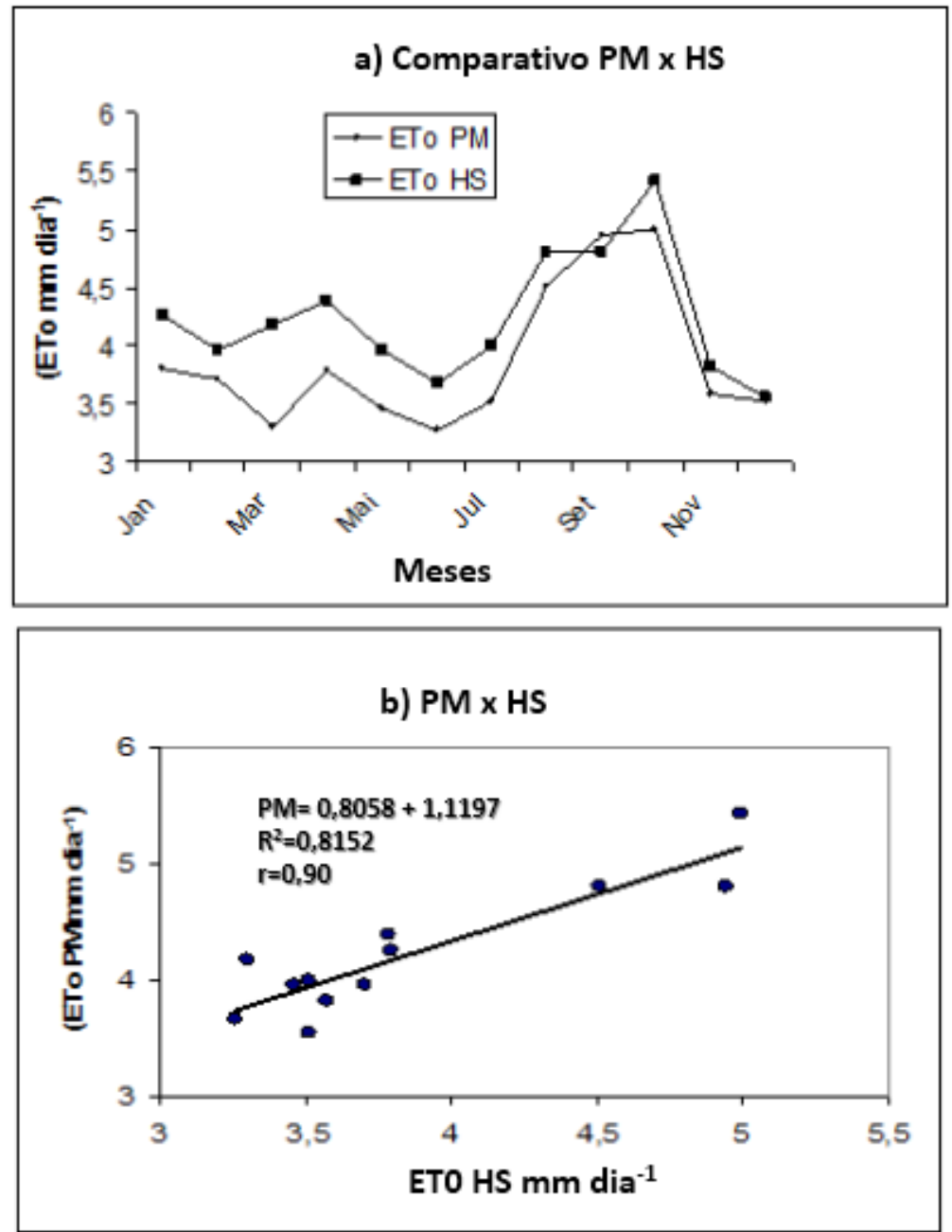

***Significativo ao nível de $0,1 \%$ de probabilidade pelo teste $\mathrm{t}$ (Student) 
Observa-se que o método de HargreavesSamani apresentou correlação alta $(r=0,90)$, em relação ao Penman-Monteith, d igual a 0,84 e c igual a 0,76, o que possibilita classificá-lo, de acordo com Camargo e Sentelhas (1997) como muito bom. O mesmo foi observado por Silva et al. (2008), quando realizaram estudo comparativo de ETO em Januária-MG, e o método HS apresentou-se muito bom, possibilitando estimativas confiáveis em todos os meses do ano.

Desta forma, na falta de dados climatológicos como radiação solar, velocidade do vento e umidade relativa, pode-se utilizar o método HS para determinação da ETO no manejo de irrigação, uma vez que necessita-se apenas da temperatura máxima e média, como parâmetros medidos, e da radiação extraterrestre, que pode ser obtida de tabelas ou de equações, utilizandose para sua obtenção apenas a data e a latitude do local.

Observa-se também que a ETO estimada por ambos os métodos tende a atingir seus valores máximos nos meses de agosto a outubro, - que pode ser explicado pelas altas temperaturas e baixa umidade do ar durante esse período, período usualmente de estiagem na região de Januária, MG.

$\mathrm{O}$ ajuste local do método de HS para a região de Januária, MG, é dado pela equação (6) resultante da regressão.

$$
\mathrm{ET}=0,8058 \mathrm{HS}+1,1197(6)
$$

em que:

$\mathrm{PM}=\mathrm{ETO}$, em $\mathrm{mm} \mathrm{dia}^{-1}$, ajustada para o método de Penman-Monteith;

$\mathrm{HS}=\mathrm{ETO}, \mathrm{em} \mathrm{mm} \mathrm{dia}{ }^{-1}$, estimada pelo método de Hargreaves-Samani;

Na Figura 2 apresenta-se a relação dos valores diários da ETO, em $\mathrm{mm} \mathrm{dia}^{-1}$, correspondentes ao método TCA e comparado ao método de PM.

Figura 2. Comparação entre a ET0 estimada por PM e TCA, referente ao período de 01 de janeiro de 2014 a 31 dezembro de 2016 (a); linha de tendência da ETO de PM e TCA, referente ao período 01 de janeiro de 2014 a 31 dezembro de 2016 (b).
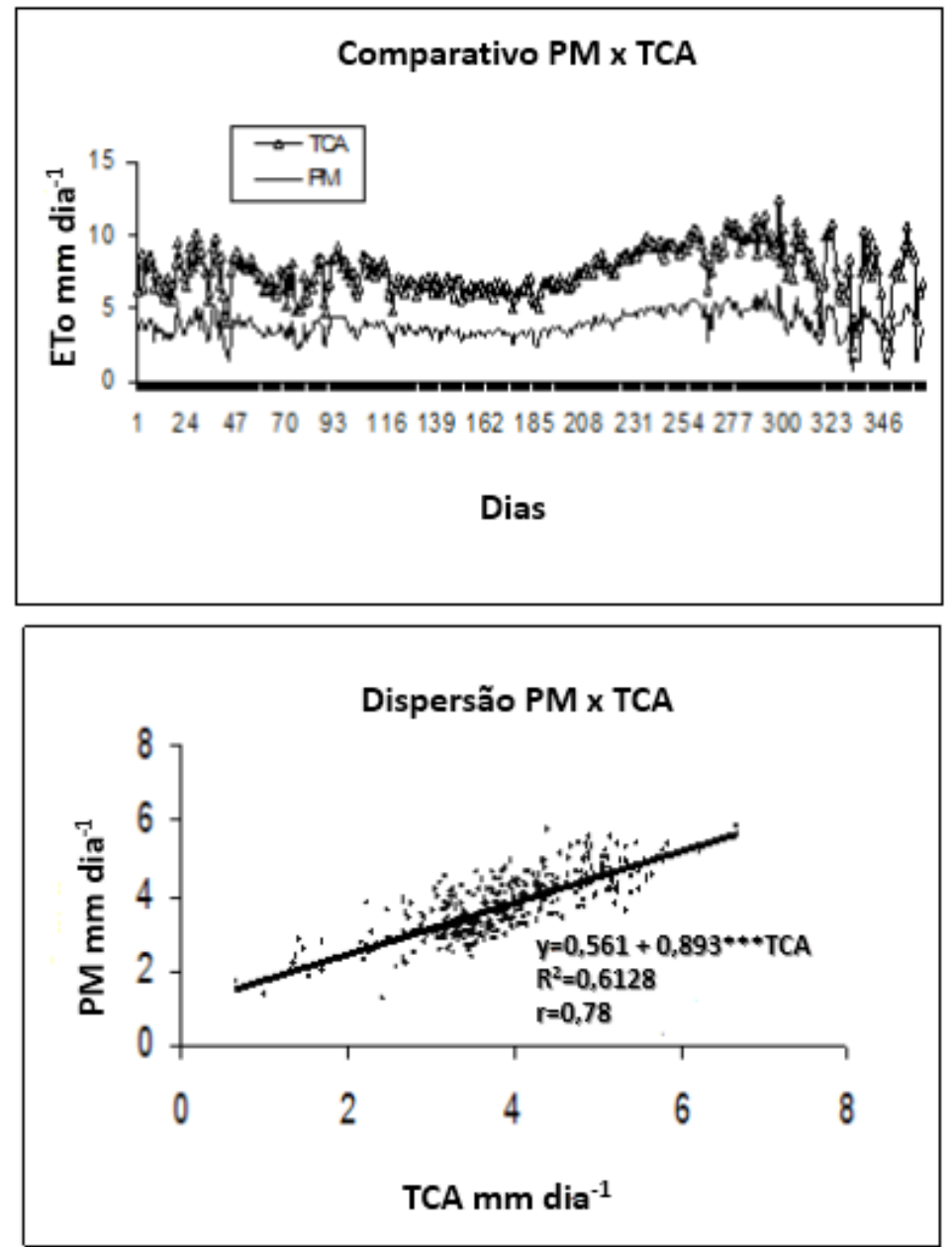

***Significativo ao nível de $0,1 \%$ de probabilidade pelo teste $\mathrm{t}$ (Student) 
Os valores de ETO obtidos pelo método TCA, durante a maioria dos dias, superestimaram a ETO obtida pelo método de PM. Resultado semelhante foram obtidos por Figueiredo et al. (2002), os quais compararam a ETO obtida pelo TCA, situado a $30 \mathrm{Km}$ da área experimental, com a ETO obtida por meio do método de PM, em projeto de irrigação do município de Jaíba, MG. Além disso, a ETO do TCA não acompanhou todas as variações que ocorreram com a evapotranspiração calculada pelo método de PM durante esse período.

O método TCA apresentou correlação muito alta $(r=0,78)$, em relação ao PenmanMonteith,

coeficiente de exatidão $(d=0,87)$ e coeficiente de desempenho $(c=0,68)$, o que possibilita classificá-lo, de acordo com Camargo \& Sentelhas (1997) como bom.

O TCA apresentou aproximação ao método de PM, com coeficiente de ajuste $\left(R^{2}\right)$ igual a 0,6128 . A equação 1 representa o ajuste do método TCA ao método de PM.

$$
\mathrm{ET}_{\mathrm{PM}}=0,561+0,893^{* * *} \mathrm{ET}_{\mathrm{TCA}}(7)
$$

em que:

$\mathrm{ET}_{\mathrm{PM}}=$ evapotranspiração ajustada para $\mathrm{O}$ método de PM, em mm dia ${ }^{-1}$

$\mathrm{ET}_{\mathrm{TCA}}=$ evapotranspiração estimada pelo método TCA, $\mathrm{mm} \mathrm{dia}^{-1}$

\section{Conclusão}

O método de Hargreaves-Samani apresentouse como eficiente, possibilitando estimativas confiáveis de evapotranspiração de referência durante todos os meses, podendo assim ser utilizado, desde que ajustado, por agricultores para o manejo de irrigação na região de JanuáriaMG. O método do TCA superestima a ETO na região de Januária, MG. Todavia o desempenho é considerado bom quando ajustado ao método de $\mathrm{PM}$, sendo assim, considerado como um método adequado para estimativa da ETO na região de Januária-MG, quando não se dispuser de todos os dados necessários para determinação pelo método de PM.

\section{Referências}

ALENCAR, C.A.B.; CUNHA, F.F.; MARTINS, C.E.; CÓSER, A.C.; ROCHA, W.S.D DA.; ARAÚJO, R.A.S. Irrigação de pastagem: atualidade e recomendações para uso e manejo. Revista Brasileira de Zootecnia, v.38, n.spe, p.98-
108, jul. 2009. http://dx.doi.org/10.1590/S1516$\underline{35982009001300012}$

ALLEN, R.G.; PEREIRA, L.S.; RAES, D.; SMITH, M. Crop evapotranspiration: Guidelines for computing crop water requirements. Rome: FAO, 1998. $300 \mathrm{p}$.

AUGUSTO, L.S.S.; GURGEL, I.G.D.; NETO, H.F.C.; MELO, C.H.; COSTA, A.M. O contexto global e nacional frente aos desafios do acesso adequado à água para consumo humano. Revista Ciência \& Saúde Coletiva, v.17, n.6, p.1511-1522, 2012. https://doi.org/10.1590/S141381232012000600015

BAUTISTA, F.; BAUTISTA, D.; CARRANZA, C.D. Calibration of the equations of Hargreaves and Thornthwaite to estimate the potential evapotranspiration in semi-arid and subhumid tropical climates for regional applications. Atmósfera, v.22, n.4, p.331-348, 2009.

BERNARDO, S. Manual de irrigação. Viçosa: UFV, 2019. $545 \mathrm{p}$.

CAMARGO, A.P.; SENTELHAS, P.C. Avaliação do desempenho de diferentes métodos de estimativa da evapotranspiração potencial no Estado de São Paulo, Brasil. Revista Brasileira de Agrometeorologia, v.5, n.1, p.89-97, 1997.

ESTEVES, B.S.; MENDONÇA, J.C.; SOUSA, E.F.; BERNARDO, S. Avaliação do Kt para estimativa da evapotranspiração de referência (ETO) em Campos dos Goytacazes, RJ. Revista Brasileira de Engenharia Agrícola e Ambiental, v.14, n.3, p.274-278, 2010.

http://dx.doi.org/10.1590/S1415$\underline{43662010000300006}$

FIGUEIREDO, F.P.; SOARES, A.A.; COSTA, L.C.; RAMOS, M.M.; OLIVEIRA, F.G. Analise comparativa das estimativas da evapotranspiração de referência, pelos métodos de Penman-Monteith e tanque classe " $A$ ", nas condições edafoclimáticas do norte de Minas Gerais. Unimontes científica, v.4, n.2, 2002.

KUNZ, J.; ÁVILA, V.S.; PETRY, M. Distribuição temporal e espacial da umidade do solo em sistemas de irrigação por gotejamento subsuperficial. Revista Monografias Ambientais, 
v.13, n.5, p.3963-3976, 2014. https://doi.org/10.5902/2236130815123

LUNARDI, J.; RABAIOLLI, J. A. Valorização e preservação dos recursos hídricos na busca pelo desenvolvimento rural sustentável. Revista OKARA: Geografia em debate, v.7, n.1, p.44-62, 2013.

MIRANDA, R.A.C.; OLIVEIRA, M.V.S.; SILVA, D.F. da. Ciclo hidrográfico planetário: abordagens e conceitos. Geo UERJ, v. 1, n. 21, p. 109-119, 2010. https://doi.org/10.12957/geoueri.2010.1461

PILAU, F.G.; BATTISTI, R.; SOMAVILLA, L.; RIGHI, E.Z. Desempenho de métodos de estimativa da evapotranspiração de referência nas localidades de Frederico Westphalen e Palmeira das Missões, RS. Ciência Rural, v.42, n.2, p.283-290, 2012. http://dx.doi.org/10.1590/S0103-

$\underline{84782012000200016}$

SILVA, E.R.; SOUZA, J.R.; SOUZA, J.A.A.; VIEIRA, G.H.S. Análise do método de Hargreaves-Samani para estimativa da evapotranspiração de referência (ETO) em Januária-MG. In: CONGRESSO NACIONAL DE IRRIGAÇÃO E DRENAGEM, 18., 2008, São Mateus. Anais [...]. São Mateus: ABID, 2008.

SILVA, V.P.R.; FILHO, A.F.B.; SILVA, B.B.; CAMPOS, J.H.B.C. Desenvolvimento de um sistema de estimativa da evapotranspiração de referência.

Revista Brasileira de Engenharia Agrícola e Ambiental, v.9, n.4, p.547-553, 2005. http://dx.doi.org/10.1590/S1415-

$\underline{43662005000400017}$

WILLMOTT, C.J.; ACKLESON, S.G.; DAVIS, R.E.; FEDDEMA, J.J.; KLINK, K.M; LEGATES, D.R.; O'DONNELL, J.; ROWE, C.M. Statistics for the evaluation and comparison of models. Journal of Geophysical Research, v.90, n.C5, p.8995-9005, 1985. https://doi.org/10.1029/JC090iC05p08995 